\title{
Grand-multiparity in Saudi Arabia-examining the obstetric risk
}

\author{
Zaheera Saadia \\ Department of Obstetrics and Gynaecology, Qassim University, Buraidah, SaudiArabia \\ Email address: \\ zaheerasaadia@hotmail.com
}

To cite this article:

Zaheera Saadia. Grand-Multiparity in Saudi Arabia_Examining the Obstetric Risk. Journal of Gynecology and Obstetrics. Vol. 2, No. 2, 2014, pp. 16-19. doi: 10.11648/j.jgo.20140202.12

\begin{abstract}
Objectives: Grand multiparity (GMP) is a term used for women who have delivered previously five or more times. Despite availability of modern obstetrical care it still remains an obstetrical risk and obstetricians consider it as a high risk pregnancy. Occurrence of GMP in developed countries has been decreasing, with the exception in some communities like Saudi Arabia. We tried to explore the relationship between obstetrical complications and grand multiparity and find out why the problem still persists despite availability of contraceptive measures. Place and duration of study: Mother and Child Hospital, Buraidah, Al-Qassim, Saudi Arabia from Jan-June 2012. Methods and Material: It was a prospective study conducted in Mother and Child hospital. Data was collected by filling a structured Proforma. GMP and women of low parity were compared by using Student t test. GMP group was asked to explain reasons for non-practicing birth spacing. SPSS-version 19 and Microsoft office 2007 for Windows 7 was used. Independent sample $t$ test was applied to compare the means and p-value below 0.05 was considered significant. Results: The average age of the GMP subjects of the study was 38.5 years, whilst the average age of the low parity women was 27.75 years. The highest occurrence of negative health outcome observed in the GMP group was anemia 51 (24.3\%) followed by preterm labor (22.3\%). Regarding the data collected on failure to use contraceptives although a number of reasons served as motivation. In order of cited prevalence, Husbands will (22.4\%) and fear of family (15.9\%) planning methods dominated. Conclusion and recommendations: There is a significant trend that points to GMP as an obstetric risk. The most cited reason for not using contraceptives in spite of their ready availability in Saudi Arabia was the husband's lack of co-operation and fear of contraceptive measures. These responses provide valuable insight into the reasons for such a high number of GMP women in Saudi Arabia and serve as the basis for important dialogue and future study.
\end{abstract}

Keywords: Grandmultipara, Anemia, Contraception

\section{Introduction}

The term 'Grand-multiparity' was introduced nearly eight decades ago by Solomon (1934), who called it the "dangerous multipara" [1]. Parity is defined as the number of times a woman has given birth to a live child, and Grand-multiparity is defined as parity equal to or greater than five previous live births [2, 3, 4, and 5]. A significant amount of research has been conducted identifying Grand-multiparity (GMP) as an independent risk factor for a variety of serious intra-partum complications (occurring during childbirth), including, placenta previa, placental abruption, fetal mal-presentation, instrumental delivery, caesarean section, postpartum haemorrhage, prematurity, newborn intensive care unit admission, and maternal death $[2,3,6,7]$.
Indeed, over the course of many decades since Solomon's initial research (which identified GMP as a significant contributing factor for obstetric risks), the perceived and actual link between GMP and obstetric complications has been ongoing, but the results of these studies have been mixed. More precisely, Solomon claimed that the risk to the mother and child is relatively high in first pregnancy, drops sharply in second and third and then slowly rises with increasing parity by the sixth pregnancy [1] Particularly, a number of studies reported notably increased risks $[2,8,9$, 10 , and 11]. On the other hand some have described only minor risks or even lower frequencies of complications among GMP's [3, 12, 13, and 14]. Recent studies have indicated that, in general, occurrence of GMP in developed countries has been steadily decreasing, with the exception of religious or cultural factors that still play a significant role in 
some populations or communities [15].

The risk of overall antenatal (pre-birth) complications increases in GMP group, with the exception of pregnancy induced hypertension (PIH), which is noted to be less frequently in that group. The author speculated that PIH was seen less frequently in their study due to age controls placed, since it is widely known that increasing age is a risk factor for hypertensive disorders [16]. Indeed, in another study conducted at Agha Khan Hospital, in Karachi, authors found advanced maternal age as the probable reason for increased incidence of PIH in their GMP group, despite the fact it was not an age-matched study [17, 18]. Additional studies have further identified other complications typically associated with grand-multiparity as independently associated with advanced maternal age $[19,20]$. Consequently, maternal age is a significant confounder that must be controlled to minimize bias in interpretation of study results.

In addition, studies have been conducted in Saudi Arabia for at least two decades, although these are not plentiful. For example a study of multiparity from Security Forces Hospital, Riyadh focused on the obstetric outcomes of patients classified as extreme multiparity (ten or more live births) in comparison to a control group of low-parity women (two to five live births). Using data from the years 1986 and 1991, the control group was comprised of 3349 women, whilst the study reviewed 228 cases of extreme multiparity. The study found higher rates of hypertension and caesarean section in the study group when compared to the control. On the other hand, no difference was found in the perinatal outcome between both groups. Interestingly, Fayed et al concluded that grand-multiparity, even to the extreme, did not pose additional obstetric or perinatal risks to mothers as long as the woman had a high socio-economic status and access to a high standard of antenatal care [21].

It is important to note, therefore, that numerous studies have explored the relationship between grand multiparity and obstetric complications but, as already noted, the result of these studies have been mixed [2]. Indeed, upon critical evaluation of these studies, it is observed that many of the populations were classified as low socio-economic status and had inadequate access to modern perinatal care, in addition to others that exhibited increased maternal age[7,22,23,24]. More recent studies seem to confirm the results of Fayed et al that grand-multiparity is not, independently, a significant obstetric risk factor as long as the mothers live in areas with high socioeconomic status and high standard perinatal care [21].

Regarding lack of contraceptive use Yasir reported a high incidence of GMP among women in Pakistan, with a resulting increase in risk for intrapartum and neonatal complications, especially when compared to women with only two or three previous live births. Unfortunately, the study did not attempt to identify reasons for the lack of contraceptive use. Interestingly the study determined that the anticipation of possible complications in conjunction with competent ante partum surveillance may improve the outcome for these patients. Thus author recommended a greater emphasis to be placed on family planning initiatives (i.e., birth control, etc.), health awareness, and delivery of quality medical care, to improve obstetric performance for women [25].

The Saudi population has one of the highest fertility rates in the world. Multiparity, grand-multiparity, and above, are seen frequently in Saudi populations, with 12 children in a family not being uncommon. However, in Bondagji's study (which took place in Bahrain), a significant factor for negative obstetric outcomes were related to unbooked deliveries (usually referring to a pregnant mother who receives no antenatal care). Yet, when the study controlled for this variable by including a similar number of unbooked deliveries in both its multiparous and GMP groups, the results "showed a significant increase in the perinatal mortality, the rate of caesarean section, medical complications and postpartum haemorrhage among (GMP) compared to multiparous women in a population with high rate of unbooked deliveries" [26].

The trend in many recent papers is toward a conclusion that the outcome of GMP is much improved when compared to previous years, especially when mothers receive adequate antenatal However, anaemia is still a common problem even in developed countries [3,27]. This could be due to some of the factors already noted, including late- or even non-booking of deliveries and low socio-economic status, or even poor spacing of children.

\section{Subjects and Methods}

It was a prospective study where women of low parity and GMP were compared. A total of 700 participants were involved in the study, with 499 classified as low parity (less than five live births) and the other 201 grand multiparous (five or more live births). The study was conducted at Mother and Child Hospital (MCH), Al-Qassim, Saudi Arabia from Jan-June 2012. MCH is a major medical facility in the region and is a tertiary care hospital with annual deliver rates of 10,000-12,000. Data was collected by completing a pro forma for each of the hospital admitted cases for delivery. Also, the GMP group was asked why they did not practice contraception (but not the low-parity group). Based on the bulk of existing literature on the topic, the null hypothesis for this study was: There is no difference between GMP and women of low parity in terms of obstetrical complications. No hypothesis was formed regarding the lack of contraceptive use, since this area has not been studied before, and no data is available to formulate a hypothesis.

To keep the data anonymous patient name or identification number was not mentioned. Proforma with incomplete data were excluded. This sample size had $95 \%$ Confidence level and a Confidence interval of 5.

SPSS-version 19 and Microsoft office 2007 for Windows 7 was used. Independent sample $t$ test was applied to compare the means and p-value below 0.05 was considered significant. 


\section{Results}

The average age of the GMP subjects of the study was 38.5 years, whilst the average age of the low parity women was 27.75 years [Fig 1]. The highest occurrence of any negative health outcome observed in the GMP group was anaemia, which was noted in 51 out of the 201 GMP women (24.3\%) as compared to (20) $4 \%$ in the low parity group [Figure 2]. In another difference identified between the two groups, 45 GMP had pre-term labor $(22.3 \%)$, whilst none of the low parity women experienced pre-term labor [Figure 2]. Pregnancy-induced hypertension (PIH) was reported in 20 $(9.95 \%)$ of the GMP group and in $13(2.6 \%)$ of the low parity group [Figure 2]. The statistical difference is once again significant. , anti-partum haemorrhage was identified in 15 of the GMP group (7.5\%), and $5(1 \%)$ in the low parity group; post-partum haemorrhage was found in 11
(5.5\%) of the GMP group and 5( $1 \%$ )of the low parity group; obstructed labor was found in 5( $2.5 \%)$ of the GMP group and none of the low parity group; and caesarean hysterectomy was noted in $5(2.5 \%)$, whilst none were observed in the low parity group and all these complications were significantly higher in GMP group [Table-1].

Regarding the data collected on failure to use contraceptives, it was revealed that a wide variety of reasons serve as motivation. In order of cited prevalence, the reasons are: Husband's will—45 (22.4\%); Fear of family planning methods-32 (15.9\%); Failure of family planning measures-29 (14.5\%); Ignorant of family planning measures-23 (11.4\%); Woman's own wish of large family size-23 (11.4\%); Religious reasons-21 (10.4\%); Trying to have a male child-15 (7.5\%); Bad obstetrical history-7 $(3.5 \%)$; Trying to have a female child-6 (3\%) [Figure- 3$)$.

Table 1. Statistical Analysis of Negative Health Outcomes (Independent sample t test).

\begin{tabular}{lllllll}
\hline Complications & Mean 1 & Variance 1 & Mean 2 & Variance 2 & t & Sig. (2-tailed) \\
\hline Anemia & 0.25 & 0.188 & 0.04 & 0.048 & 0.000 \\
Preterm labour & 0.22 & 0.175 & 0.02 & 0.020 & 0.000 \\
PIH & 0.10 & 0.090 & 0.05 & 0.048 & 2.418 \\
APH & 0.07 & 0.069 & 0.01 & 0.01 & 4.708 \\
PPH & 0.05 & 0.048 & 0.01 & 0.01 & 3.305 \\
Obstructed labour & 0.02 & 0.024 & 0.00 & 0.00 & 0.000 \\
Cesarean hysterectomy & 0.02 & 0.024 & 0.00 & 0.00 & 3.563 \\
\hline
\end{tabular}

P-value $<0.05=$ Very strong evidence against the null hypothesis

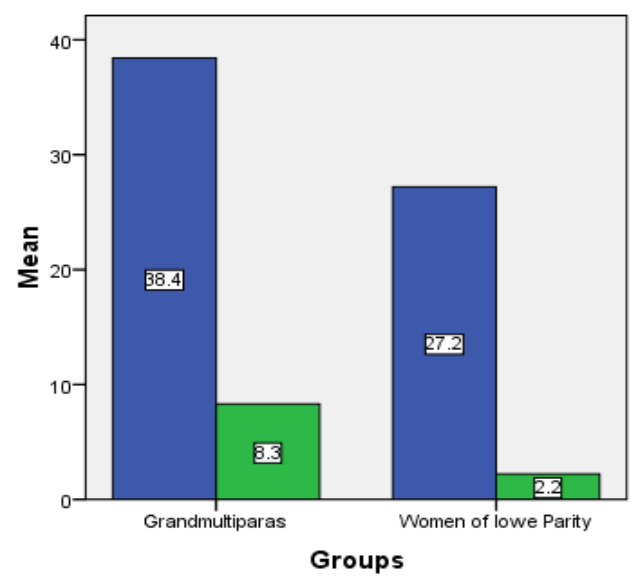

Figure 1. Average age and parity among both groups.

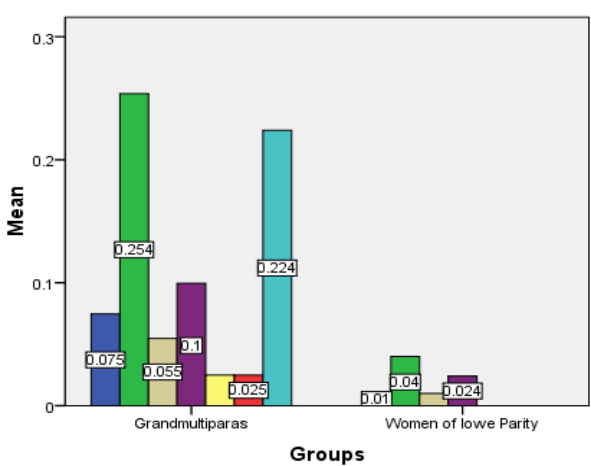

Figure 2. Mean values of negative health outcome in both groups.

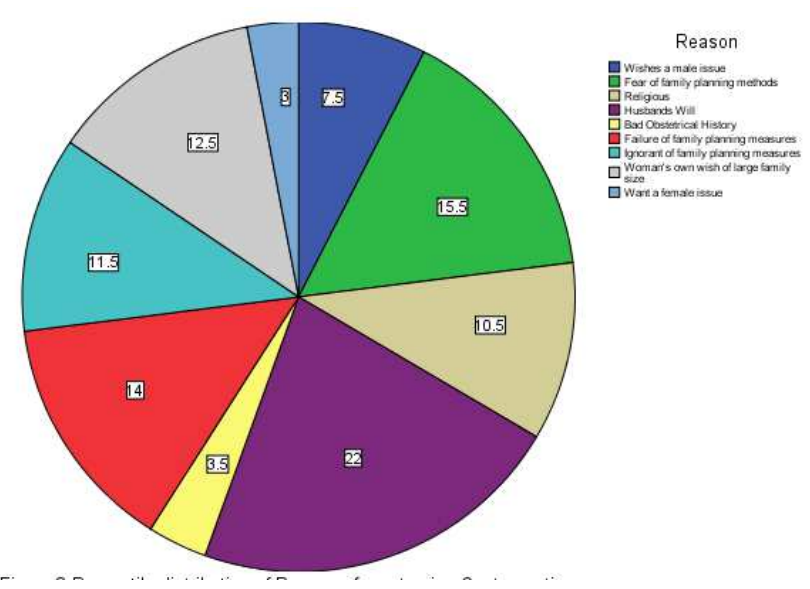

Figure 3. Percentile distribution of Reasons for not using Contraceptives.

\section{Acknowledgements}

The author acknowledges the help and support of $\mathrm{MCH}$ staff.

\section{References}

[1] Solomon, B. 1934. The dangerous multipara. Lancet 2: 8-11.

[2] Babinszki A, Kerenyi T, Torok O, Grazi V, Lapinski RH, Berkowitz RL. Perinatal outcome in grand and great-grand multiparity: effects of parity on obstetric risk factors. Am J Obstet Gynecol. 1999 Sep;181(3):669-74. 
[3] Bugg GJ, Atwal GS, Maresh M. Grandmultiparae in a modern setting. BJOG. 2002 Mar;109(3):249-53

[4] Al Rowaily M A, Abolfotouh MA. Predictors of gestational diabetes mellitus in a high parity community in Saudi Arabia. EMHJ. 2010; 16(6). Retrieved from, http://applications.emro.who.int/emhj/V16/06/16_6_2010_0 636_0641.pdf

[5] Opara EI, Zaidi J. The interpretation and clinical application of the word 'parity': a survey. BJOG. 2007 Oct;114(10):1295-7.

[6] Simonsen SM, Lyon JL, Alder SC, Varner MW. Effect of grand multiparity on intrapartum and newborn complications in young women. Obstet Gynecol. 2005 Sep;106(3):454-60.

[7] Roman H, Robillard PY, Verspyck E, Hulsey TC, Marpeau L, Barau G. Obstetric and neonatal outcomes in grand multiparity. Obstet Gynecol. 2004 Jun;103(6):1294-9.

[8] Fuchs K, Peretz BA, Marcovici R, Paldi E, Timor-Tritsh I. The "grand multipara"--is it a problem? A review of 5785 cases. Int J Gynaecol Obstet. 1985 Sep; 23(4):321-6.

[9] Hughes PF, Morrison J. Grandmultiparity--not to be feared? An analysis of grandmultiparous women receiving modern antenatal care. Int J Gynaecol Obstet.1994 Mar;44(3): 211-7.

[10] Seidman DS, Dollberg S, Stevenson DK, Gale R. The effects of high parity and socioeconomic status on obstetric and neonatal outcome. Arch Gynecol Obstet.1991; 249(3):119-27

[11] Tanbo TG, Bungum L. The grand multipara--maternal and neonatal complications. Acta Obstet Gynecol Scand. 1987;66(1):53-6. PubMed PMID: 3604593.

[12] Abu-Heija AT, Chalabi HE. Great grand multiparity: is it a risk? J Obstet Gynaecol. 1998 Mar;18(2):136-8.

[13] Kaplan B, Harel L, Neri A, Rabinerson D, Goldman GA, Chayen B. Great grand multiparity--beyond the 10th delivery. Int J Gynaecol Obstet. 1995 Jul;50(1):17-9.

[14] Lyrenäs S. Labor in the grand multipara. Gynecol Obstet Invest.2002;53(1):6-12.

[15] Severinski NS, Mamula O, Severinski S, Mamula M. Maternal and fetal outcomes in grand multiparous women. Int J Gynaecol Obstet. 2009 Oct;107(1):63-4

[16] Yasmeen L, Rasheed, T. \& Syed, S. Is Grandmultiparity Still a Risk Factor for Obstetric Complications? Annals of Pakistan Institute of Medical Sciences. 2010; 6(1): 58-61.

[17] Munim S, Rahbar MH, Rizvi M, Mushtaq N. The effect of grandmultiparity on pregnancy related complications: the Aga Khan University experience. J Pak Med Assoc. 2000 Feb;50(2):54-8.

[18] Eidelman AI, Kamar R, Schimmel MS, Bar-On E. The grandmultipara: is she still a risk? Am J Obstet Gynecol. $1988 \mathrm{Feb} ; 158(2): 389-92$.

[19] Bobrowski RA, Bottoms SF. Underappreciated risks of the elderly multipara. Am J Obstet Gynecol. 1995 Jun;172(6):1764-7; discussion 1767-70.

[20] Chan BC, Lao TT. Effect of parity and advanced maternal age on obstetric outcome. Int J Gynaecol Obstet. 2008 Sep;102(3):237-41. Epub 2008 Jul 7. PubMed PMID: 18606410.

[21] Fayed HM, Abid SF, Stevens B. Risk factors in extreme grand multiparity. Int $\mathbf{J}$ Gynaecol Obstet. 1993 Apr;41(1):17-22.

[22] Ezra Y, Schmuel E, Hakim M, Schenker JG. The outcome of grand-multiparous pregnancies of Arabic and Jewish populations in peripheral and central areas of Israel. Acta Obstet Gynecol Scand. 2001 Jan;80(1):30-3.

[23] Kumari AS, Badrinath P. Extreme grandmultiparity: is it an obstetric risk factor? Eur J Obstet Gynecol Reprod Biol. 2002 Feb; 101(1): 22-5.

[24] Shamshad B. Age and parity related problems affecting outcome of labor in grandmultiparous. Pakistan Journal of medical sciences. 2004: 42(4):179-184

[25] Yasir R. Grandmultiparity-Still an obstetric risk for developing country. Channel. 2010; 16(2: 264-267

[26] Bondagji, N. The Perinatal and Neonatal Outcome in Grand-Grand Multiparous Women, A Comparative Case Control Study. Bahrain Medical Bulletin . 2005; 27(4): 1-5.

[27] Nordin NM, Fen CK, Isa S, Symonds EM. Is grandmultiparity a significant risk factor in this new millennium? Malays J Med Sci. 2006 Jul;13(2):52-60.

[28] Kalaivani K. Prevalence \& consequences of anaemia in pregnancy. Indian J Med Res. 2009 Nov;130(5):627-33. Review. 